Revista PSICOLOGIA, 2015, Vol. 29 (1), 35-44

\title{
Relações entre Adaptabilidade de carreira e personalidade: Um estudo com universitários ingressantes brasileiros
}

\author{
Marucia Patta Bardagi ${ }^{1}$, Patricia Albanaes ${ }^{1}$ \\ ${ }^{1}$ Universidade Federal de Santa Catarina (UFSC), Brasil
}

\begin{abstract}
Resumo: Identificar os fatores que influenciam a experiência universitária dos estudantes é importante para a pesquisa no desenvolvimento de carreira e permite uma maior compreensão da adaptação acadêmica dos alunos. Este estudo investigou as relações entre traços de personalidade e adaptabilidade de carreira em universitários ingressantes brasileiros. 359 alunos participaram (59.3\% mulheres), com idade média de 20.6 anos, que responderam a perguntas biodemográficas, uma escala de adaptabilidade de carreira e uma lista de marcadores de personalidade. Foram encontradas correlações significativas entre adaptabilidade e todas as dimensões da personalidade. Não houve diferenças significativas entre os sexos na adaptabilidade e os estudantes de Humanidades tiveram escores significativamente mais baixos em preocupação de carreira do que alunos de outras áreas. Os resultados favorecem a discussão sobre o impacto das características de entrada dos estudantes para a adaptação e o engajamento acadêmico.
\end{abstract}

Palavras-chave: Adaptabilidade de carreira; Personalidade; Estudantes universitários.

Relationship between career adaptability and personality: A study with Brazilian freshmen university students: Identify factors that influence students' university experience is important to research in career development and allows a greater understanding of the students' academic adaptation. This study investigated relations between personality traits and career adaptability in Brazilian university freshmen. 359 students participated (59.3\% women) with a mean age of 20.6 years, who responded to biodemographic questions, a career adaptability scale, and a list of personality traits. Significant correlations were found between adaptability and all personality dimensions. There were no significant gender differences in adaptability, and students of Humanities had significantly lower scores in career concern than students from other areas. The results incite discussions on the impact of students' incoming characteristics for adaptation and academic engagement.

Keywords: Career adaptability; Personality; University students.

Identificar fatores que favoreçam o envolvimento acadêmico e aumentem a probabilidade de permanência dos alunos no ensino superior não é importante apenas nos âmbitos vocacional ou econômico. Desde os estudos clássicos de Berger (1994), Pascarella e Terenzini (2005), mas também nos mais recentes (Martins \& Ferreira, 2011), têm-se evidências de que a permanência no ensino superior é também um potencializador do desenvolvimento pessoal dos indivíduos, além de provocar mudanças de atitudes e valores, de qualidade de vida, aumentar o poder econômico e promover o desenvolvimento de carreira. No Brasil, em um estudo avaliando a experiência universitária e sua influência sobre o desenvolvimento pessoal dos alunos, Pachane (2004) descreveu que a maioria dos participantes graduandos em uma instituição do Estado de São Paulo - relatou aumento na autoconfiança (89.34\%), independência (86.89\%) e responsabilidade (81.96\%), maior visão de mundo (81.15\%), aumento da sociabilidade $(79.51 \%)$, melhora no autoconceito $(72.13 \%)$ e mudanças significativas nos valores (57.38\%). De forma geral, $72.95 \%$ achavam que o fato de estarem na universidade tem relação direta com as mudanças ocorridas em si mesmo.

Embora os benefícios da vivência acadêmica no ensino superior sejam claros, há um grande contingente de alunos que tem uma experiência negativa com a entrada na universidade. Esta experiência pode ser para alguns um processo tranquilo, mas para outros ser geradora de angústias e acompanhada, inclusive, de estados depressivos, conforme revisão de Santos e Almeida (2002). Os índices de evasão e o aumento na busca de estudantes universitários por atendimento em serviços de carreira confirmam que a transição para o ensino superior é um momento de vulnerabilidade e pode ser vivenciada como crise por

${ }^{1}$ Dados de contacto para correspondência: Marucia Bardagi, Laboratório de Informação e Orientação Profissional da UFSC - sala 14ª , Departamento de Psicologia, Centro de Filosofia e Ciências Humanas UFSC. Campus João David Ferreira Lima, bairro Trindade, Florianópolis/SC. CEP 88040-900. E-mail: marucia.bardagi@gmail.com 
muitos estudantes.

Nesse sentido, no âmbito do desenvolvimento de carreira, além da importância da formação e do impacto da educação superior para o desenvolvimento futuro dos alunos, outras linhas de investigação focam seus esforços na identificação da qualidade da experiência universitária e de fatores que promovem uma melhor adaptação e engajamento acadêmicos dos alunos. Exemplos são os estudos sobre as vivências acadêmicas (Almeida \& Soares, 2004; Sousa, Bardagi, \& Nunes, 2013), a autoeficácia (Bardagi \& Boff, 2010; Ourique \& Teixeira, 2012; Samssudin \& Barros, 2011), a adaptabilidade de carreira (Guan et. al., 2013; Saldanha, 2013; Silveira, 2013; Scholl \& Cascone, 2011), a satisfação e qualidade de vida (Diener \& Diener, 2009; Lounsbury, Saudargas, Gibson, \& Leong, 2005; Soares, Pereira, \& Canavarro, 2014), a personalidade (Faria, 2013; Ourique \& Teixeira, 2012), entre outros. Este estudo concentra-se nos constructos da adaptabilidade e da personalidade.

Com relação à personalidade, sabe-se que há diferentes formas de entendê-la e de avaliar as características individuais das pessoas. Uma das mais frequentes na literatura recente em Psicologia é o Modelo dos Cinco Grandes Fatores (Big Five) (McCrae \& John, 1992), versão moderna da Teoria de Traço que representa um avanço conceptual e empírico no campo da personalidade, descrevendo dimensões humanas básicas de forma consistente e passível de replicação. 0 modelo é composto por cinco dimensões denominadas neuroticismo (predisposição para experimentar afetos negativos), extroversão (sociabilidade e assertividade), abertura (disposição para experimentar sentimentos, novas ideias e flexibilidade), socialização (altruísmo, cuidado, confiança e cooperação) e realização (persistência, necessidade de alcançar objetivos, responsabilidade).

A avaliação das relações entre personalidade e desenvolvimento de carreira dos indivíduos ao longo do ciclo vital tem sido destaque em pesquisas de diferentes modelos teóricos no âmbito vocacional. No contexto do ensino superior têm sido conduzidos, por exemplo, estudos apontando as relações entre as dimensões do Big Five e interesses vocacionais (Valentini, Teodoro, \& Balbinotti, 2009), habilidades sociais dos alunos (Bartholomeu, Nunes, \& Machado, 2008; Bueno, Sales Oliveira, \& Oliveira, 2001), motivação acadêmica (Komarraju, Karau, \& Schmeck, 2009), bem estar subjetivo (Nunes, Hutz, \& Giacomoni, 2009), decisão e planejamento de carreira (Gunkel \& Schlaegel, 2010; Ourique \& Teixeira, 2012) e desempenho (Poropat, 2009). Estes estudos são consistentes em apontar o papel preditivo das características de personalidade sobre as variáveis de interesse e indicar as relações positivas entre as dimensões extroversão, abertura, realização e socialização e os resultados mais favoráveis ao desenvolvimento do aluno, bem como as relações negativas entre neuroticismo e boa adaptação ou ajustamento.

O estudo de Lounsbury et al., (2005), especificamente, encontrou correlações entre satisfação de vida e universitária com todas as dimensões do Big Five, que explicaram $45 \%$ da variância da medida de satisfação geral, à exceção de neuroticismo, que se correlacionou negativamente com as medidas de satisfação; os autores concluem que quem os alunos se tornam na universidade e quão satisfeitos eles serão com os diferentes aspectos da experiência universitária depende prioritariamente de quem eles são quando ingressam no Ensino Superior. Faria (2013), encontrou correlações negativas significativas entre neuroticismo e reações afetivas à exploração de carreira (satisfação com a informaç̧ão) e correlações positivas com as dimensões stress na exploração e stress na decisão. 0 estudo de Schnuck e Handal (2011) investigou junto a 190 universitários ingressantes, a relação entre estilo parental, personalidade e adaptação à universidade. Dentre os resultados encontrados destacam-se que o neuroticismo foi associado negativamente com todas as variáveis de adaptação universitária, e os traços de extroversão, socialização e realização foram associados significativamente à adaptação positiva. 0 estudo de metaanálise de Poropat (2009), sobre as relações entre personalidade e desempenho, que reuniu mais de 70000 casos em amostras de estudantes dos diferentes níveis educacionais concluiu que a performance dos alunos se correlaciona positiva e significativamente com socialização, realização e abertura.

Quanto aos estudos brasileiros referentes à relação entre personalidade e diferentes aspectos ligados à experiência universitária, Bueno et al., (2001) encontraram correlações significativas entre habilidades sociais e traços de personalidade segundo o modelo dos cinco grandes fatores. Na mesma linha de pesquisa, Bartholomeu, Nunes e Machado (2008) encontraram correlações significativas entre habilidades sociais em discentes e traços de socialização de acordo com o modelo Big Five, além de diferenças significativas entre os sexos, em que as mulheres apresentaram uma maior incidência de socialização. Já Ourique e Teixeira (2012) encontraram correlação positiva entre extroversão e realização com o planejamento de carreira dos universitários, e correlação negativa entre planejamento e a dimensão neuroticismo.

0 segundo aspecto enfatizado neste estudo e que tem recebido atenção recente dos pesquisadores do desenvolvimento de carreira é a adaptabilidade. Super e Knasel (1981) propuseram o termo adaptabilidade de carreira para enfocar o equilíbrio que cada indivíduo busca entre o mundo do trabalho 
e a sua vida pessoal. Deste modo, os autores deslocaram "a atenção da maturidade de carreira como prontidão para tomada de decisões para a adaptabilidade de carreira como prontidão para lidar com mudanças no trabalho e em suas condições" (Super \& Knasel, 1981, p. 83). 0 termo responde a um contexto em que sujeitos adultos tomam inúmeras decisões de carreira - tanto voluntárias quanto forçadas. A adaptabilidade de carreira é um constructo psicológico que se refere à capacidade dos indivíduos para gerenciar suas carreiras frente ao cenário de mudanças e incertezas que caracterizam o mundo do trabalho contemporâneo. Enquanto alguns se indivíduos mostram capazes de lidar com as incertezas, outros apresentam dificuldades para responder às demandas de autonomia, comportamento exploratório, tomada de decisão e planejamento profissional (Lassance, 2005).

De acordo com o modelo de adaptabilidade de carreira descrito por Savickas (2013), este constructo é composto por quatro dimensões: preocupação, controle, curiosidade e confiança. A preocupação está relacionada com a orientação ao futuro e ao planejamento no que diz respeito à carreira. 0 controle refere-se às crenças do indivíduo acerca da sua responsabilidade frente ao futuro e à sua vontade de agir proativamente em prol de seus objetivos. A curiosidade relaciona-se a comportamentos de caráter exploratório que permitem ao indivíduo ampliar suas perspectivas de ação no cenário ocupacional. Por fim, a confiança refere-se às crenças de capacidade para enfrentar os desafios relacionados com a construção da carreira. Assim, conforme Silveira (2013), indivíduos adaptáveis seriam aqueles capazes de se preocuparem e possuírem um senso de controle sobre seu futuro vocacional, de lançarem mão da curiosidade para explorarem possíveis identidades e cenários futuros e de fortalecerem a confiança para alcançarem suas aspirações. Nesse sentido, níveis altos de adaptabilidade de carreira poderiam indicar boas condições de lidar com os desafios da transição para a universidade, aumentar o engajamento acadêmico dos alunos e, potencialmente, contribuir para a permanência e satisfação dos mesmos.

Nos estudos internacionais, a adaptabilidade é bastante estudada em amostras de indivíduos adultos, já inseridos no mercado de trabalho ou em situação de desemprego. Pesquisas com amostras universitárias mostram, por exemplo, que a adaptabilidade tem sido positivamente correlacionada com autoeficácia (Guan et al., 2013) e pode ser potencializada através de intervenções de aconselhamento com estudantes (Scholl \& Cascone, 2011). Há ainda poucos estudos brasileiros acerca da adaptabilidade. Silva e Teixeira (2013), por exemplo, realizaram um estudo que buscou compreender de que forma as experiências de estágio contribuem para o desenvolvimento da adaptabilidade de carreira. Os resultados indicaram que, embora todas as dimensões de adaptabilidade de carreira influenciem a qualidade das vivências acadêmicas e sejam por elas influenciadas, as dimensões curiosidade e confiança pareceram estar mais relacionadas com os benefícios do estágio e à construção das bases para a transição ao papel profissional. Outras pesquisas com estudantes universitários (Silveira, 2013; Teixeira, Lassance, \& Bardagi, 2011) confirmam as relações positivas entre adaptabilidade e características de personalidade como abertura a experiência, realização e extroversão, apontadas em estudos internacionais (Rottinghaus, Day, \& Borgen, 2005). Ainda, foi observada no estudo brasileiro de Teixeira et al., (2011) uma correlação positiva entre adaptabilidade e satisfação de vida em universitários. 0 principal instrumento de medida do constructo adaptabilidade de carreira - o Career Adapt-Ability Inventory - foi adaptado e validado para a população brasileira (Teixeira, Bardagi, Duarte, Lassance, \& Magalhães, 2012) numa iniciativa da equipe brasileira do Life Design International Research Group (Savickas et al., 2009).

Com o intuito de contribuir com o crescente número de estudos sobre o desenvolvimento vocacional do estudante universitário no Brasil, com foco em variáveis que a literatura tem descrito como influentes na adaptação, satisfação e sucesso acadêmico, esta pesquisa buscou identificar as relações entre características de personalidade e adaptabilidade de carreira, entre alunos ingressantes do ano de 2013 de uma instituição pública federal do sul do Brasil. Como os estudos sobre adaptabilidade e personalidade conduzidos no país tiveram como participantes alunos em fases mais avançadas dos cursos (Silveira, 2013; Teixeira et al., 2011), considera-se que os resultados desta investigação podem ser úteis na identificação dos perfis gerais dos alunos ingressantes e também na possível identificação de perfis de estudantes com maior vulnerabilidade à evasão (baixos índices de adaptabilidade combinados com baixos índices de realização, socialização e abertura e altos índices de neuroticismo, por exemplo).

Ainda, pretendeu-se identificar eventuais diferenças de gênero e área de formação presentes na amostra. Com isso, será possível contribuir com informações que poderão servir, no futuro, para a elaboração de estratégias de intervenção voltadas para os alunos ingressantes com foco na potencialização do engajamento acadêmico e na permanência no ensino superior. Com base na revisão da literatura, espera-se que a adaptabilidade se correlacione com as dimensões de extroversão, abertura, socialização e realização de forma positiva e com neuroticismo de forma negativa. 


\section{MÉTODO}

\section{Participantes}

Participaram do estudo 359 graduandos (59.3\% do sexo feminino e $40.7 \%$ do sexo masculino), com idades entre 18 e 59 anos $(M=20.6 ; D P=5.22)$, sendo $90 \%$ desses com idades entre 18 e 25 anos. Do total, $94.2 \%$ eram solteiros, $5.3 \%$ são casados ou em união estável. Apenas 3.9\% possuíam filhos. A escolaridade dos pais dos participantes está distribuída da seguinte forma: 19\%, 28.8\% e $52.2 \%$ das mães e $21.5 \%, 30.3 \%$ e $48.2 \%$ dos pais frequentaram o ensino fundamental, médio e superior, respectivamente. A renda familiar estimada era em $35.3 \%$ dos casos de até 2.500 reais (cerca de 790 Euros 2 ), em $24 \%$ dos casos entre 2.501 e 4.500 reais (de 790 a 1420 Euros), $16.7 \%$ das participantes possuíam renda familiar entre 4.501 e 7.000 reais (de 1420 a 2220 Euros), outros $8.2 \%$ possuíam renda estimada entre 7.001 e 9.500 reais (2220 a 3010 Euros), e $15.8 \%$ possuem renda superior a 9.500 reais (acima de 3010 Euros).

A maioria da amostra também se caracterizou por um sistema de ingresso regular na universidade (74.4\%), e outros $25.6 \%$ dos participantes ingressaram na instituição pelo sistema de cotas. Esse sistema é parte de uma política de ações afirmativas no Ensino Superior no Brasil (Domingues, 2005), que reserva parte das vagas na universidade para alunos oriundos da rede pública de ensino e, dentre estes, alunos negros ou índios.

Os participantes da pesquisa participavam de atividades acadêmicas em $46.4 \%$ dos casos, sendo essas relativas à equipe esportiva (17.5\%), estágio (35.5\%), extensão (22.9\%), monitoria (16.3\%) e pesquisa (34.3\%). Embora $20.1 \%$ dos sujeitos estejam inseridos no mercado de trabalho, a maioria desses $(64.9 \%)$ indicou que o trabalho não estava vinculado à área de estudos. Ao ingressarem na universidade, $75.5 \%$ indicaram o curso atual como aquele de sua preferência, enquanto $24.5 \%$ o apontaram como não preferencial. No que diz respeito às relações entre área de formação e inserção no mercado de trabalho, observou-se maior frequência de alunos trabalhadores na área de Ciências Sociais Aplicadas e de alunos que apenas estudam nas áreas de Engenharias e Ciências Agrárias $\left(\chi^{2}=-2.4 ; g l=7\right.$; $p<.001$ ). Quanto à participação em atividades de integração (informativas, curriculares ou sociais) promovidas no primeiro semestre de ingresso à universidade, a maioria (98.3\%) indicou ter participado de ao menos uma delas.

Os dados da pesquisa foram analisados estatisticamente, com auxílio do pacote SPSS 20.0. Inicialmente, além da estatística descritiva (composta também pelos testes de associação Qui-quadrado), foram realizados testes $t$ de diferença de médias, correlações de Pearson e análises de variância para compreender as relações entre as variáveis.

\section{Instrumentos e Procedimentos}

Foi utilizado para a coleta de dados um questionário online com acesso restrito aos participantes e aos responsáveis pelo estudo, composto pelos seguintes instrumentos:

a) Ficha de dados biossociodemográficos, com o objetivo de caracterizar a amostra e identificar alguns aspectos relevantes da inserção acadêmica, incluindo a participação em atividades de integração;

b) Inventário de Adaptabilidade (CAAS Brasil) (Teixeira et al., 2012): 24 itens que avaliam as quatro dimensões da adaptabilidade - preocupação, controle, curiosidade e confiança, com sete itens cada, respondidos numa escala tipo Likert de cinco pontos. Os resultados obtidos por meio do cálculo do coeficiente Alpha de Cronbach neste estudo indicam índices de consistência interna satisfatórios: adaptabilidade total $(\alpha=.90)$, preocupação $(\alpha=.81)$, confiança $(\alpha=.77)$, curiosidade $(\alpha=.80)$, e controle $(\alpha=.82)$.

c) Marcadores de Personalidade (Hutz et al., 1998): os Adjetivos Marcadores da Personalidade foram desenvolvidos por Hutz et al. (1998) como um instrumento para mensurar de forma breve os Cinco Grandes Fatores de Personalidade - Socialização, Extroversão, Realização, Neuroticismo e Abertura. A partir de listas de adjetivos desenvolvidas na literatura internacional, e após análise fatorial, os autores identificaram 64 adjetivos e obtiveram uma solução fatorial coerente com a expectativa de cinco fatores. Os fatores identificados (e o respectivo número de adjetivos) são os seguintes: Fator I - Socialização (16), Fator II - Extroversão (10), Fator III - Realização (14), Fator IV - Neuroticismo (12) e Fator V - Abertura (12). A pontuação final é obtida através da média das respostas dadas aos itens de cada fator. 0 uso dos marcadores se deveu à maior agilidade de preenchimento e menor tamanho do instrumento em comparação com outros disponíveis para a avaliação do Big Five (Nunes, Hutz, \& Nunes, 2008). Neste estudo, os índices de consistência interna foram todos superiores a 79.

Com a disponibilização da lista de contatos dos estudantes ingressantes do primeiro semestre de 2013 da instituição pela Superintendência de Governança Eletrônica e Tecnologia da Informação e Comunicação (SeTIC), mediante autorização institucional, foram enviados e-mails para os alunos com o

${ }^{2}$ Cotações em euros feitas em março de 2015. 
link para preenchimento dos instrumentos. A coleta de dados ocorreu entre os meses de outubro e dezembro do ano de 2013. 0 questionário online foi enviado para 2573 alunos ingressantes e respondido por 359 graduandos, uma taxa de $13.95 \%$ de retorno. 0 projeto foi submetido e aprovado pelo Comitê de Ética em pesquisa (no CAAE 03287213.3.0000.0121), seguiu todas as indicações éticas para a pesquisa com seres humanos (Brasil, 2012) e fez parte de um estudo maior apoiado pelo CNPq através do edital Ciências Humanas 2011 (chamada MCTI /CNPq /MEC/CAPES N o 07/2011).

\section{RESULTADOS}

Quanto aos aspectos de personalidade e adaptabilidade de carreira, o Quadro 1 indica as médias e desvios padrão dos níveis das quatro dimensões da adaptabilidade de carreira (confiança, controle, curiosidade, preocupação) de acordo com área de formação, sexo e sistema de ingresso na universidade (regular ou cotas), além da amostra total.

Quadro 1. Médias $(M)$ e desvios-padrão $(D P)$ dos níveis das quatro dimensões da adaptabilidade de carreira, de acordo com área, sexo e sistema de ingresso na instituição.

\begin{tabular}{|c|c|c|c|c|c|c|c|c|c|c|}
\hline & \multicolumn{2}{|c|}{ Confiança } & \multicolumn{2}{|c|}{ Controle } & \multicolumn{2}{|c|}{ Curiosidade } & \multicolumn{2}{|c|}{ Preocupação } & \multicolumn{2}{|c|}{$\begin{array}{c}\text { Adaptabilidade } \\
\text { total }\end{array}$} \\
\hline & $M$ & $D P$ & $M$ & $D P$ & $M$ & $D P$ & $M$ & $D P$ & $M$ & $D P$ \\
\hline \multicolumn{11}{|l|}{ Área de formação } \\
\hline Ciências Agrárias & 23.81 & 4.28 & 24.32 & 4.37 & 21.74 & 4.20 & 23.65 & 4.18 & 93.52 & 14.55 \\
\hline Ciências Biológicas & 22.46 & 2.18 & 22.69 & 4.09 & 21.31 & 3.17 & 23.62 & 3.73 & 90.08 & 6.44 \\
\hline Ciências da Saúde & 23.07 & 4.32 & 22.93 & 4.51 & 20.68 & 4.27 & 23.78 & 4.42 & 90.46 & 13.27 \\
\hline Ciências Exatas e da Terra & 23.00 & 4.22 & 22.93 & 4.51 & 20.68 & 4.27 & 23.78 & 4.42 & 88.55 & 15.69 \\
\hline Ciências Humanas & 23.21 & 5.00 & 21.08 & 5.34 & 21.15 & 5.03 & 20.72 & 6.23 & 86.15 & 17.01 \\
\hline Ciências Sociais Aplicadas & 23.74 & 4.01 & 23.33 & 4.85 & 21.84 & 4.45 & 23.71 & 4.05 & 92.62 & 13.27 \\
\hline Engenharias & 23.29 & 3.95 & 23.41 & 4.11 & 21.57 & 3.99 & 22.56 & 4.33 & 90.84 & 12.88 \\
\hline Linguística. Letras e Artes & 23.55 & 3.58 & 22.27 & 4.25 & 21.82 & 2.96 & 22.58 & 3.81 & 90.21 & 12.13 \\
\hline \multicolumn{11}{|l|}{ Sexo } \\
\hline Feminino & 23.06 & 4.13 & 22.78 & 4.68 & 21.09 & 4.27 & 23.02 & 4.42 & 89.95 & 13.63 \\
\hline Masculino & 23.78 & 4.00 & 23.16 & 4.54 & 21.79 & 4.30 & 22.64 & 4.64 & 91.37 & 14.04 \\
\hline \multicolumn{11}{|l|}{ Sistema de ingresso } \\
\hline Cotas & 23.62 & 4.18 & 23.02 & 4.80 & 21.28 & 4.49 & 23.38 & 4.27 & 91.30 & 13.90 \\
\hline Regular & 23.26 & 4.06 & 22.91 & 4.57 & 21.40 & 4.23 & 22.69 & 4.59 & 90.26 & 13.78 \\
\hline Amostra total & 23.35 & 4.09 & 22.94 & 4.62 & 21.37 & 4.29 & 22.87 & 4.51 & 90.53 & 13.80 \\
\hline
\end{tabular}

Já o Quadro 2 indica os mesmos índices dos cinco fatores dos Marcadores de Personalidade, também em relação à área, sexo, sistema de ingresso e para a amostra total.

Quadro 2. Médias $(M)$ e desvios-padrão $(D P)$ dos marcadores de personalidade, de acordo com área, sexo e sistema de ingresso na instituição.

\begin{tabular}{|c|c|c|c|c|c|c|c|c|c|c|}
\hline & \multicolumn{2}{|c|}{ Abertura } & \multicolumn{2}{|c|}{ Extroversão } & \multicolumn{2}{|c|}{ Neuroticismo } & \multicolumn{2}{|c|}{ Realização } & \multicolumn{2}{|c|}{ Socialização } \\
\hline & $M$ & $D P$ & $M$ & $D P$ & $M$ & $D P$ & $M$ & $D P$ & $M$ & $D P$ \\
\hline \multicolumn{11}{|l|}{ Área de formação } \\
\hline Ciências Agrárias & 55.71 & 9.29 & 44.55 & 12.58 & 34.81 & 11.32 & 71.39 & 10.27 & 82.52 & 11.55 \\
\hline Ciências Biológicas & 55.62 & 7.81 & 47.85 & 10.96 & 38.69 & 12.30 & 69.31 & 12.67 & 83.15 & 10.92 \\
\hline Ciências da Saúde & 54.05 & 9.75 & 47.73 & 10.25 & 35.68 & 10.47 & 70.98 & 11.15 & 83.15 & 13.63 \\
\hline Ciências Exatas e da Terra & 54.43 & 9.65 & 44.00 & 12.94 & 35.31 & 14.65 & 68.21 & 12.40 & 79.10 & 13.13 \\
\hline Ciências Humanas & 56.38 & 11.13 & 44.67 & 12.40 & 37.46 & 10.43 & 65.46 & 15.82 & 79.13 & 15.46 \\
\hline Ciências Sociais Aplicadas & 57.08 & 9.40 & 44.90 & 13.10 & 37.53 & 11.25 & 71.23 & 12.41 & 83.10 & 14.77 \\
\hline Engenharias & 55.84 & 8.20 & 43.30 & 13.89 & 39.95 & 11.78 & 68.93 & 10.47 & 79.78 & 12.52 \\
\hline Linguística. Letras e Artes & 60.61 & 9.06 & 47.42 & 11.93 & 38.73 & 11.46 & 69.18 & 11.39 & 84.45 & 13.02 \\
\hline \multicolumn{11}{|l|}{ Sexo } \\
\hline Feminino & 55.62 & 10.08 & 46.79 & 12.39 & 36.14 & 10.53 & 70.15 & 12.15 & 83.25 & 13.51 \\
\hline Masculino & 57.05 & 8.19 & 42.42 & 12.68 & 37.25 & 13.17 & 68.36 & 11.78 & 78.88 & 13.04 \\
\hline \multicolumn{11}{|l|}{ Sistema de ingresso } \\
\hline Cotas & 57.65 & 9.63 & 45.99 & 11.92 & 36.86 & 12.51 & 68.38 & 11.48 & 83.35 & 13.22 \\
\hline Regular & 55.70 & 9.25 & 44.68 & 12.93 & 36.50 & 11.39 & 69.78 & 12.20 & 80.83 & 13.52 \\
\hline Amostra total & 56.20 & 9.37 & 45.01 & 12.67 & 36.59 & 11.67 & 69.42 & 12.02 & 81.48 & 13.47 \\
\hline
\end{tabular}


Não houve diferenças significativas nas médias entre sexo e sistema de ingresso em relação aos níveis de adaptabilidade de carreira. Por outro lado, no que se refere à área de formação, os estudantes de Ciências Humanas apresentaram menor média nos níveis de preocupação com a carreira em relação aos alunos das demais áreas, $F(7,358)=2.11 ; p<0.05$. Quanto aos aspectos de personalidade, as mulheres apresentaram níveis significativamente maiores de extroversão e socialização em relação aos homens, $t=$ -3.24; $g l=357, p<0.01 ; t=-3.05 ; g l=357 ; p<0.01$, respectivamente; não houve diferenças significativas nas médias de personalidade entre alunos cotistas e não cotistas nem em relação às áreas de formação.

0 Quadro 3 concerne às correlações entre as variáveis de interesse do estudo. Quanto à renda familiar ou idade, não houve relações significativas com as dimensões de adaptabilidade ou os fatores de personalidade.

Quadro 3. Correlações entre o Inventário de Adaptabilidade e os Marcadores de Personalidade.

\begin{tabular}{|c|c|c|c|c|c|c|c|c|c|}
\hline Variáveis & 1 & 2 & 3 & 4 & 5 & 6 & 7 & 8 & 9 \\
\hline 1. Adaptabilidade total & - & & & & & & & & \\
\hline 2. Preocupação & $.75^{*}$ & - & & & & & & & \\
\hline 3. Confiança & $.78^{*}$ & $.43^{*}$ & - & & & & & & \\
\hline 4. Controle & $.83^{*}$ & $.51^{*}$ & $.54^{*}$ & - & & & & & \\
\hline 5. Curiosidade & $.78^{*}$ & $.39^{*}$ & $.53^{*}$ & $.54^{*}$ & - & & & & \\
\hline 6. Extroversão & .19 & $.87^{*}$ & $.24^{*}$ & .16 & .14 & - & & & \\
\hline 7. Realização & $.56^{*}$ & $.46^{*}$ & $.34^{*}$ & $.60^{*}$ & $.33^{*}$ & -.02 & - & & \\
\hline 8. Neuroticismo & $-.31^{*}$ & -.10 & $-.35^{*}$ & $-.32^{*}$ & -.19 & $-.43^{*}$ & $-.21^{*}$ & - & \\
\hline 9. Abertura & $.36^{*}$ & .15 & $.29^{*}$ & $.29^{*}$ & $.42^{*}$ & .18 & $.23^{*}$ & -.07 & - \\
\hline 10. Socialização & $.28^{*}$ & $.22^{*}$ & .14 & $.31^{*}$ & .18 & .09 & $.39^{*}$ & $-.24^{*}$ & $.34^{*}$ \\
\hline
\end{tabular}

" $\mathrm{p}<0.001$

A análise do Quadro 3 permite verificar a ocorrência de correlações significativas entre a adaptabilidade total e suas dimensões e todas as dimensões de personalidade. Quanto às relações entre personalidade e adaptabilidade, de maior magnitude são as correlações entre extroversão e preocupação com a carreira, realização e controle e realização e preocupação com a carreira. A dimensão neuroticismo correlacionouse negativamente com a adaptabilidade total e com confiança e controle. Como esperado, as dimensões de adaptabilidade se correlacionam entre si, bem como as dimensões de personalidade, ambas na direção indicada pela literatura.

\section{DISCUSSÃO E CONSIDERAÇÕES FINAIS}

Esse estudo teve como objetivo identificar as relações entre adaptabilidade de carreira e características de personalidade em estudantes ingressantes universitários. A seguir serão destacados alguns aspectos centrais relacionados com os resultados encontrados na pesquisa.

Inicialmente, quanto às informações sociodemográficas, observa-se a inexistência de correlações entre sexo ou área de formação e a participação em atividades no curso, como já indicado em outras pesquisas (Bardagi \& Boff, 2010). Entretanto, significativa parcela dos discentes não apresentou envolvimento com práticas acadêmicas. 0 desenvolvimento e aprimoramento de espaços que privilegiem a inserção de alunos em atividades extracurriculares é uma necessidade institucional que pode contribuir com o engajamento em atividades exploratórias, descritas na literatura como impactantes na qualidade da trajetória acadêmica e na permanência dos alunos no Ensino Superior (Bardagi \& Boff, 2010; Fior \& Mercuri, 2009). Nesse sentido, apesar de pouco envolvidos com atividades acadêmicas, é importante o resultado indicando que a quase totalidade dos alunos se engajou em atividades de acolhimento ao ingressante, que costumam servir como espaços para descobrir as possibilidades oferecidas pela instituição e também como oportunidades de inserção inicial dos alunos em atividades extracurriculares, também favorecendo a adaptação. Defende-se que o desenvolvimento e o constante aprimoramento das atividades voltadas para a adaptação do estudante ao Ensino Superior - sejam de cunho social, informativo ou vocacional - pode aumentar o nível de conforto dos alunos na transição para a universidade, fazendo-se necessária a permanente reflexão sobre o desenvolvimento e a avaliação de estratégias que visem alcançar esses objetivos.

Parece merecer atenção, no âmbito vocacional, a informação de que $24.5 \%$ dos alunos participantes não estão inseridos no seu curso de preferência. Considera-se que esse elemento possa influenciar nas experiências acadêmicas iniciais desses sujeitos, podendo haver um engajamento acadêmico inicial inferior em relação aos demais, além de uma maior vulnerabilidade à evasão. É possível supor também que esse aspecto seja efeito, em parte, das atuais políticas públicas no Brasil de fomento à inserção no ensino superior, como a adoção do sistema de cotas, por exemplo. Essas políticas são mecanismos de ação afirmativa que objetivam a reserva de vagas para grupos minoritários. Nesse 
sentido, para essa parcela da população que historicamente foi alvo de exclusão social, o interesse pela entrada no Ensino Superior pode ser preponderante em relação à reflexão sobre a escolha do curso compatível com a preferência pessoal. Entretanto, a médio e longo prazo, a não identificação com o curso e área de formação, tanto para cotistas quanto não cotistas, pode trazer prejuízos à integração, satisfação, motivação e desempenho (Bardagi, Lassance, Paradiso, \& Menezes, 2006; Pachane, 2004) devido às divergências percebidas entre características e planos pessoais e características da profissão a ser seguida.

Ao analisarem-se os índices de adaptabilidade e personalidade da amostra total e de acordo com algumas características demográficas e de formação, é interessante observar a relativa uniformidade nos níveis de adaptabilidade, considerando-se área de formação, sexo e sistema de ingresso dos alunos. Esses resultados sugerem que esses sujeitos apresentam uma linearidade favorável em relação às condições vocacionais para lidar com os desafios da transição para a universidade e para se adaptarem à mesma. Especificamente, os resultados referentes à não diferença entre sexos é, em certa medida, incongruente com os resultados obtidos anteriormente em amostras brasileiras, que apresentaram maiores índices de controle entre os homens (Silveira, 2013), ou maiores índices de preocupação de carreira entre as mulheres (Teixeira et al., 2011). Como a literatura internacional também apresenta resultados inconsistentes em relação às diferenças de sexo na adaptabilidade, sugerem-se novos estudos que busquem investigar esta relação, trazendo maior compreensão sobre os processos de carreira de homens e mulheres.

Outro aspecto a ser ponderado é que talvez as diferenças na adaptabilidade apareçam com maior força à medida que a formação avança, o contato com a realidade profissional aumenta e as próprias capacidades e limitações são testadas ao longo do curso. A forma como cada área promove estratégias de formação e preparação dos alunos para o campo de trabalho pode ter influência na adaptabilidade futura dos alunos. Estudos comparando os níveis de adaptabilidade de alunos de diferentes áreas de estudo ao longo da formação podem trazer luz a estas questões.

Quanto à ausência de diferença na adaptabilidade entre cotistas e não cotistas, este é um resultado congruente com estudos que indicam também a semelhança no desempenho acadêmico (Velloso, 2009) e na autoeficácia (Sousa, Bardagi, \& Nunes, 2013) nesses dois grupos. Nesse sentido, este estudo é mais um resultado que se contrapõe à expectativa de que os alunos cotistas apresentam piores índices de desempenho e também de adaptação à universidade (Sousa et al., 2013; Velloso, 2009).

Não houve também diferenças significativas entre os níveis de adaptabilidade total e área de formação. Entretanto, especificamente na dimensão preocupação com a carreira, alunos de Ciências Humanas apresentaram menores escores em comparação com discentes das demais áreas. 0 indivíduo que carece de recursos no âmbito da dimensão Preocupação apresenta geralmente uma atitude de indiferença face à carreira, revelando maior apatia, pessimismo e falta de planejamento em comparação àqueles com maiores índices de preocupação, conforme Savickas (2013). Ao se considerar essa faceta como essencial no desenvolvimento de carreira dos estudantes, a diferença encontrada em relação aos índices de preocupação entre estudantes de Ciências Humanas e as demais áreas carece de maior investigação. Embora não tenham sido encontradas na literatura vocacional avaliações relacionadas a esta questão, futuras pesquisas com foco mais específico nesse elemento podem fornecer maior consistência a estas relações, bem como subsidiar ações institucionais em direção ao favorecimento do envolvimento acadêmico dos alunos da área de Humanidades.

Nos fatores de personalidade, houve diferenças significativas quanto ao sexo. As mulheres apresentaram maiores índices de socialização, em convergência com os resultados encontrados por Bartholomeu, Nunes e Machado (2008) e extroversão - indicada pela literatura como relacionada significativamente com as variáveis planejamento de carreira e comportamento exploratório (Ourique \& Teixeira, 2012). Aqui se pode pensar em uma maior disponibilidade feminina para o estabelecimento de relações interpessoais e exploração de carreira, o que pode gerar uma maior adaptação das mulheres ao ambiente acadêmico e os seus novos atores (colegas, professores, técnicos, etc) tanto quanto favorecer o engajamento nas atividades acadêmicas e a identificação com a profissão.

Os resultados também apontam para a relação teoricamente estabelecida entre adaptabilidade e todas as dimensões de personalidade (Rottinghaus et al., 2005; Silveira, 2013; Teixeira et al., 2011). De maior magnitude são as correlações entre extroversão e preocupação com a carreira, realização e controle e realização e preocupação com a carreira. A literatura é bastante consistente em indicar a correlação significativa entre realização e o desenvolvimento profissional (Ourique \& Teixeira, 2012). Nessa direção, a dimensão realização, referente à persistência, responsabilidade com a carreira e necessidade de obtenção de resultados, configura-se como aspecto destacável no que tange aos comportamentos que influenciam significativamente no desenvolvimento vocacional dos estudantes universitários. Baixos índices dessa dimensão podem impactar negativamente nesse momento de 
transição para a Educação Superior, sendo possivelmente experienciado de forma excessivamente ansiogênica pelo sujeito, levando a prejuízos tanto no que se refere à sua adaptação acadêmica quanto ao seu bem-estar psicológico.

Em contrapartida, a dimensão neuroticismo correlacionou-se negativamente com a adaptabilidade total e com confiança e controle. A literatura também aponta a correlação negativa entre esta faceta e planejamento e decisão de carreira (Ourique \& Teixeira, 2012), satisfação de vida universitária (Lounsbury et al., 2005), comportamento exploratório (Faria, 2013; Reed et al., 2004), satisfação na carreira (Boudreau et al., 2001) e definição da identidade vocacional (Tokar \& Swanson, 1995). Além disso, traços de neuroticismo foram identificados como fator impactante negativamente na adaptação universitária (Schnuck \& Handal, 2011). Tais resultados são essenciais na identificação de perfis de estudantes com maior vulnerabilidade. Nesse sentido, considera-se que baixos níveis de adaptabilidade combinados com baixos índices de realização e altos índices de neuroticismo podem ser relacionados a uma maior dificuldade no envolvimento, satisfação e adaptação universitária. Assim, para indivíduos com predisposição a afetos negativos e baixos índices de adaptabilidade de carreira, a transição para o Ensino Superior pode constituir-se como um maior desafio em relação à sua adaptação acadêmica. E estes estudantes podem ser os maiores beneficiados por intervenções de carreira com foco na adaptabilidade (Scholl \& Cascone, 2011).

Por fim, é importante apontar que o presente estudo apresenta algumas limitações. Inicialmente, algumas áreas de formação tiveram uma maior representação estudantil, em relação a outras áreas. É possível supor que, essa não equivalência na quantidade de participantes das diversas áreas pode ter impactado nas diferenças existentes entre essa variável e as dimensões de adaptabilidade de carreira existentes. Outra limitação refere-se à incipiência da avaliação da personalidade através dos marcadores de personalidade. Nesse sentido, Hutz et al. (1998) afirmam que esse modelo não corresponde a um sistema explicativo completo, sendo que uma avaliação global da personalidade deve levar em consideração também as características únicas de cada sujeito, além do contexto na avaliação dos fatores. Outros estudos com amostras mais homogêneas e que façam uma avaliação de personalidade de forma mais completa poderão trazer elementos para complementar as reflexões aqui apontadas.

\section{Referências}

Almeida, L. S., \& Soares, A. P. (2004). Os estudantes universitários: Sucesso escolar e desenvolvimento psicossocial. In E. Mercuri \& S. A. J. Polydoro (Eds.), Estudante universitário: Características e experiências de formação (pp. 15-40). Taubaté, SP: Cabral Editora e Livraria Universitária.

Bardagi, M. P., \& Boff, R. M. (2010). Autoconceito, autoeficácia profissional e comportamento exploratório em universitários concluintes. Avaliação, 15, 41-56.

Bardagi, M. P., Lassance, M. C., Paradiso, A., \& Menezes, L. (2006). Escolha profissional e inserção no mercado de trabalho: Percepções de estudantes formandos. Psicologia Escolar e Educacional, 10, 69-82.

Bartholomeu, D., Nunes, C. H. S. S., \& Machado, A. A. (2008). Traços de personalidade e habilidades sociais em universitários. Psico-USF, 13, 41-50.

Berger, K. S. (1994). The developing person through the life span. EUA: Warth Publishers.

Boudreau, J. W., Boswell, W. R., \& Judge, T. A. (2001). Effects of personality on executive career success in the United States and Europe. Journal of Vocational Behavior, 58, 53-81.

Bueno, J. M. H., Sales Oliveira, S. M. S., \& Oliveira, J. C. S. (2001). Um estudo correlacional entre habilidades sociais e traços de personalidade. Psico-USF, 6, 31-38.

Conselho Nacional de Saúde (Brasil). Resolução n 466, de 12 de dezembro de 2012 [PDF]. Retirado de http://www.conselho.saude.gov.br/web_comissoes/conep/index.html.

Diener, E., \& Diener, M. (2009). Cross-cultural correlates of life satisfaction and self-esteem. In E. Diener (Ed.), Culture and well-being: The collected works of Ed Diener (pp. 71-91). Netherlands: Springer.

Domingues, P. (2005). Ações afirmativas para negros no Brasil: 0 início de uma reparação histórica. Revista Brasileira de Educação, 29, 164-176.

Faria, L. C. (2013). Personalidade e reações afetivas à exploração de carreira. Psicologia Ciência e Profissão, 33, 100-111.

Fior, C. A., \& Mercuri, E. (2009). Formação universitária e flexibilidade curricular: Importância das atividades obrigatórias e não obrigatórias. Psicologia da Educação. 29, 191-215.

Guan, Y., Deng, H., Sun, J., Wang, Y., Cai, Z., Ye, L., Fu, R., Wang, Y., Zhang, S., \& Li, Y. (2013). Career adaptability, job search self-efficacy and outcomes: A three-wave investigation among Chinese University Graduates. Journal of Vocational Behavior, 83, 561-570. 
Gunkel, M., \& Schlaegel, C. (2010). The influence of personality on students' career decisiveness - A comparison between Chinese and German economics and management students. Management revue, $21,229-243$.

Hutz, C. S., Nunes, C. H., Silveira, A. D., Serra, J. G., Anton, M. C., \& Wieczorek, L. S. (1998). 0 desenvolvimento de marcadores para a avaliação da personalidade no Modelo dos Cinco Grandes Fatores. Psicologia: Reflexão e Crítica, 11, 395-415.

Komarraju, M., Karau, S. J., \& Schmeck, R. R. (2009). Role of the Big Five personality traits in predicting college students' academic motivation and achievement. Learning and Individual Differences, 19, 47-52.

Lassance, M. C. P. (2005). Adultos com dificuldades de ajustamento ao trabalho: Ampliando o enquadre da orientação vocacional de abordagem evolutiva. Revista Brasileira de Orientação Profissional, 6, 41-51.

Lounsbury J. W., Saudargas, R. A., Gibson, L. W., \& Leong, F. T. (2005). An investigation of broad and narrow personality traits in relation to general and domain-specific life satisfaction of college students. Research in Higher Education, 46, 707-729.

Martins, E. C. F., \& Ferreira, J. A. G. (2011). Evolução do pensamento, raciocínio e desempenho em alunos do ensino superior. Revista Portuguesa de Educação, 24, 213-246.

McCrae, R. R., \& John, O. P. (1992). An introduction to the Five-Factor Model and its applications. Journal of Personality, 60, 175-216.

Nunes, C. H. S. S., Hutz, C. S., \& Giacomoni, C. H. (2009). Associação entre bem estar subjetivo e personalidade no modelo dos cinco grandes fatores. Avaliação Psicológica, 8, 99-108.

Nunes, C. H. S. S., Hutz, C. S., \& Nunes, M. F. (2008). Bateria Fatorial de Personalidade. São Paulo: Casa do Psicólogo.

Ourique, L. R., \& Teixeira, M. A. P. (2012). Autoeficácia e personalidade no planejamento de carreira de universitários. Psico-USF, 17, 311-321.

Pachane, G. G. (2004). A experiência universitária e sua contribuição ao desenvolvimento pessoal do aluno. In: E. Mercuri \& S. A. J. Polydoro (Eds.), Estudante universitário: Características e experiências de formação (pp.155-186). Taubaté, SP: Cabral Ed. e Livraria Universitária.

Pascarella, E. T., \& Terenzini. P. T. (2005). How college affects students: A third decade of research. San Francisco, CA: Jossey-Bass.

Poropat, A. E. (2009). A meta-analysis of the five-factor model of personality and academic performance. Psychological Bulletin, 135, 322-338.

Reed, M. B., Bruch, M. A., \& Haase, R. F. (2004). Five-factor model of personality and career exploration. Journal of Career Assessment, 12, 223-238.

Rottinghaus, P., Day, S., \& Borgen, F. (2005). The career futures inventory: A measure of career-related adaptability and optimism. Journal of Career Assessment, 13, 3-24.

Saldanha, M. B. C. (2013). Adaptabilidade de carreira em trabalhadores-estudantes do ensino superior. (Dissertação de mestrado). Universidade Federal do Rio Grande do Sul, Porto Alegre, RS, Brasil.

Samssudin, S., \& Barros, A. (2011). Relação entre as crenças de auto-eficácia e o apoio social na transição para o trabalho em estudantes finalistas do ensino superior. Psicologia, 25, 159-171.

Santos, L., \& Almeida, L. S. (2002). Vivências e rendimento académicos: A integração dos alunos na Universidade. In A. S. Pouzada, L. S. Almeida \& R. M. Vasconcelos (Eds.). Contextos e dinâmicas da vida académica (pp. 127-136). Guimarães: Universidade do Minho.

Savickas, M. L. (2013). Career construction theory and practice. In R. W. Lent \& S. D. Brown (Eds.), Career development and counseling: Putting theory and research to work (pp. 147-183). Hoboken, New Jersey: John Wiley \& Sons.

Savickas, M. L., Nota, L., Rossier, J., Dauwalder, J. P., Duarte, M. E.,Guichard, J., Soresi, S., Van Esbroeck, R., \& Van Vianen, A. E. M. (2009). Life-designing: A paradigm for career construction in the 21th century. Journal of Vocational Behavior, 75, 239-250.

Schnuck, J., \& Handal, P. J. (2011). Adjustment of college freshmen as predicted by both perceived parenting style and the five factor model of personality and adjustment. Psychology, 2, 275- 278.

Scholl, M. B., \& Cascone, J. (2011). The constructivist résumé: Promoting the career adaptability of graduate students in counseling programs. Career Development Quarterly, 59, 180-191.

Silva, C. S. C., \& Teixeira, M. A. P. (2013). Experiências de estágio: Contribuições para a transição universidade-trabalho. Paidéia, 23, 103-112.

Silveira, A. A. (2013). Escala de adaptabilidade de carreira: Evidências de validade e fidedignidade em uma amostra de universitários brasileiros (Dissertação de mestrado não publicada). Universidade Federal do Rio Grande do Sul, Porto Alegre, RS, Brasil. 
Soares, A. M., Pereira, M., \& Canavarro, J. P. (2014). Saúde e qualidade de vida na transição para o ensino superior. Psicologia, Saúde \& Doenças, 15, 356-379.

Sousa, H., Bardagi, M. P., \& Nunes, C. H. S. S. (2013). Autoeficácia na formação superior e vivências de universitários cotistas e não cotistas. Avaliação Psicológica, 12, 253-261.

Super, D. E., \& Knasel, E. G. (1981). Career development in adulthood: Some theoretical problems. British Journal of Guidance and Counseling, 9, 194-201.

Teixeira, M. A. P., Bardagi, M. P., Duarte, M. E., \& Magalhães, M. O. (2012). Career adaptabilities scalebrazilian form: Psychometric properties and relationships to personality. Journal of Vocational Behavior, 80, 680-685.

Teixeira, M. A. P., Lassance, M. C. P., \& Bardagi, M. P. (2011). Adaptabilidade de carreira em estudantes universitários: Efeitos do sexo e da situação de trabalho. Resumo. Anais do V Congresso brasileiro de avaliação psicológica. São Paulo: Casa do Psicólogo.

Tokar, D. M., \& Swanson, J. L. (1995). Evaluation of the correspondence between Holland's Vocational Typology and the Five-Factor Model of Personality. Journal of Vocational Behavior, 46, 89-108.

Valentini, F., Teodoro, M. L. M., \& Balbinotti, M. A. A. (2009). Relações entre interesses vocacionais e fatores de personalidade. Revista Brasileira de Orientação Profissional, 10, 57-68.

Velloso, J. (2009). Cotistas e não-cotistas: Rendimento de alunos da Universidade de Brasília. Cadernos de Pesquisa, 39, 621-644.

Historial do artigo

Recebido 20/10/2014

Aceite $\quad 03 / 04 / 2015$

Publicado 06/2015

Apoio à publicação: Fundação para a Ciência e a Tecnologia (Ministério da Educação e Ciência, Portugal) - Programa FACC 